\title{
Influence of causal language on causal understanding: A comparison between Swiss German and Turkish
}

\author{
Ebru Ger ${ }^{\mathrm{a}, 1, *}$, Larissa Stuber $^{\mathrm{a}}$, Aylin C. Küntay ${ }^{\mathrm{b}}$, Tilbe Göksun ${ }^{\mathrm{b}}$, Sabine Stoll ${ }^{\mathrm{c}, \mathrm{d}, 2}$, \\ Moritz M. Daum a,d,e,2 \\ ${ }^{a}$ Department of Psychology, University of Zurich, 8050 Zurich, Switzerland \\ ${ }^{\mathrm{b}}$ Department of Psychology, Koç University, 34450 Sariyer/İstanbul, Turkey \\ ${ }^{\mathrm{c}}$ Department of Comparative Language Science, University of Zurich, 8050 Zurich, Switzerland \\ ${ }^{\mathrm{d}}$ Center for the Interdisciplinary Study of Language Evolution (ISLE), 8032 Zurich, Switzerland \\ e Jacobs Center for Productive Youth Development, University of Zurich, 8050 Zurich, Switzerland
}

\section{A R T I C L E I N F O}

\section{Article history:}

Received 18 December 2020

Revised 8 April 2021

Available online 21 June 2021

\section{Keywords:}

Causal language

Causal understanding

Children

Causative constructions

Crosslinguistic

Lexical

Morphological

\begin{abstract}
A B S T R A C T
Young children have difficulties in understanding untypical causal relations. Although we know that hearing a causal description facilitates this understanding, less is known about what particular features of causal language are responsible for this facilitation. Here, we asked two questions. First, do syntactic and morphological cues in the grammatical structure of sentences facilitate the extraction of causal meaning? Second, do these different cues influence this facilitation to different degrees? We studied children learning either Swiss German or Turkish, two languages that differ in their expression of causality. Swiss German predominantly uses lexical causatives (e.g., schniidä [cut]), which lack a formal marker to denote causality. Turkish, alongside lexical causatives, uses morphological causatives, which formally mark causation (e.g., ye [eat] vs. yeDIr [feed]). We tested 2.5- to 3.5-year-old children's understanding of untypical cause-effect relations described with either noncausal language (e.g., Here is a cube and a car) or causal language using a pseudo-verb (e.g., lexical: The cube gorps the car).
\end{abstract}

\footnotetext{
* Corresponding author.

E-mail address: ebruger@gmail.com (E. Ger).

${ }^{1}$ During the work of her dissertation, Ebru Ger was a fellow of the "International Max Planck Research School on the Life Course" (IMPRS LIFE; http://www.imprs-life.mpg.de; http://www.imprs-life.mpg.de/participating institutions: Max Planck Institute for Human Development, Free University of Berlin, Humboldt University of Berlin, University of Michigan, University of Virginia, University of Zurich).

${ }^{2}$ Sabine Stoll and Moritz M. Daum share the senior authorship.
} 
We tested 135 Turkish-learning children (noncausal, lexical, and morphological conditions) and 90 Swiss-German-learning children (noncausal and lexical conditions). Children in both language groups performed better in the causal language condition(s) than in the noncausal language condition. Furthermore, Turkishlearning children's performance in both the lexical and morphological conditions was similar to that of Swiss-German-learning children in the lexical condition and did not differ from each other. These findings suggest that the structural cues of causal language support children's understanding of untypical causal relations regardless of the type of construction.

(C) 2021 The Authors. Published by Elsevier Inc. This is an open access article under the CC BY license (http://creativecommons.org/ licenses/by/4.0/).

\section{Introduction}

Imagine a person pulling a chair to sit on, which results in the chair moving. Presumably, it is as easy for a young child to perceive the cause-effect relation in this event as it is for an adult. Now, imagine a toddler crying over a melting ice cream on a sunny warm day. Although it is clear to an adult that the heat from the sun is causing the ice cream to melt, it might be a complete mystery to the toddler. Describing such hard-to-grasp cause-effect relations with causal language has been shown to help young children perceive these causal relations (Bonawitz et al., 2010; Butler \& Markman, 2012; Muentener, Bonawitz, Horowitz, \& Schulz, 2012). In this study, we asked which features of causal language might be responsible for boosting children's understanding of untypical causal relations. We investigated whether children learning Turkish or Swiss German use language-specific cues to uncover these causal relations.

There are several elements that render causal events typical and easy to perceive as causal. First, physical contact between a cause and an effect is an important factor in the perception of causality (Carey \& Spelke, 1994; Leslie, 1993; Michotte, 1963). Even 6-month-old infants perceive that a moving object causes a stationary object to launch when the former contacts the latter, but not when there is no contact (Cohen \& Amsel, 1998; Kotovsky \& Baillargeon, 2000; Leslie, 1984; Leslie \& Keeble, 1987). Second, dispositional agents, which are capable of acting intentionally, are key for infants in attributing causal powers. Although infants perceive inanimate objects as causers of other objects' launching of motion, they do not expect them to be causers in many other types of events. For example, only dispositional agents such as a hand and a self-propelled entity, but not inert objects, are perceived as capable of picking up things (Leslie, 1984) or changing the state of objects such as breaking them apart (Muentener \& Carey, 2010). Infants also expect moving inert objects to have been launched by a dispositional agent (Saxe, Tenenbaum, \& Carey, 2005; Saxe, Tzelnic, \& Carey, 2007).

To make sense of the world, and to make accurate predictions and interventions, children need to understand not only typical but also untypical causal relations that lack the prototypical causality features "direct physical contact" and "dispositional agents." For instance, the toddler in our example will need to learn that the sun is the causer of the ice cream's melting, although it is an inanimate causer and there is no visible physical contact. The toddler can then make sense of why ice cream melts on sunny days and can predict the same to happen on another sunny day. Moreover, if someone knows a causal relation to exist between two events, that person can intervene on the cause event to bring about the effect event (Gopnik et al., 2004; Woodward, 2007). Having learned the causal relation of heat and melting, our toddler in principle should be able to bring the ice cream close to another heat source to melt it. Children develop the inference of causal relations over the first few years of life. At 4 years, children make these causal interventions upon observing inanimate agents causing distal outcomes; however, at 2 years, toddlers do not yet do so (Bonawitz et al., 2010; Meltzoff, Waismeyer, \& Alison, 2012). 
The use of causal language by adults is effective in bootstrapping young children's understanding of untypical causal relations (Bonawitz et al., 2010; Butler \& Markman, 2012; Muentener et al., 2012). In the study of Bonawitz et al. (2010), children first observed an untypical causal event involving an inanimate agent (i.e., an object) and lacking direct physical contact between the cause and effect. A block moved on its own toward a base and touched it, and then a remote toy helicopter (connected to the base only with a wire) lighted up and started to spin. Thereafter, children were invited to act on the block to activate the toy with the prompt "Now it's your turn. Can you make it go?" Whereas preschoolers (mean age of 47 months) moved the block to contact it with the base and looked expectantly to the toy, toddlers (mean age of 24 months) did so only when the experimenter had initially described the event with causal language such as "The block can make the toy go" but not when the experimenter used noncausal language such as "See the block? Here it goes!" Moreover, when hearing the causal description "The block makes it go," toddlers performed similarly regardless of whether they were prompted with the same words ("Can you make it go?") or with different wording ("Can you turn it on?"). Thus, the effect of causal language was robust to changes in the wording when the meaning remained the same. A follow-up study using an occluded causal inference paradigm (Muentener et al., 2012) demonstrated that causal language did not merely help toddlers to initiate the causal intervention (i.e., moving the block toward the base to activate the toy) but already helped them to represent the initial predictive relation as causal (i.e., expecting contact between the block and the base when the toy activates but expecting a gap when the toy does not activate).

When the causal relations are not given in isolation but rather are given in complex scenes, causal language has been shown to help preschoolers as well (Butler \& Markman, 2012). Children aged 4 years were presented with a toy lion that laughed when it picked up one of two plastic animals and said "Hmm" when it picked up the other animal. In this simple scenario, children were equally likely to infer the causal relation whether or not they heard a causal description before the presentation (e.g., "Some animals make Lion laugh"). However, in a more complex scene where some distractor items were picked up but did not elicit a laugh or a "Hmm," the causal description improved children's causal inferences. A causal description that excluded the agents but maintained the causal relation (e.g., "Some things make Lion laugh") similarly helped. This consolidated the inference that the expression of causality (i.e., to make Lion laugh) facilitates causal understanding in complex causal scenarios.

The evidence suggests that hearing causal language helps children to understand untypical causal relations or causal evidence embedded in complex scenes. One possible mechanism that supports this is directing children's attention to the causal relation and helping them to connect the otherwise disparate representation of single events into a causal unity. However, we still do not know what particular feature of causal language is responsible for this facilitative effect. More specifically, are the semantics of verbs or phrases such as "turn on" and "make it go" crucial, or is the structure of causal language such as syntactic and morphological cues sufficient? Previous studies focused only on English and used only a single type of causative construction, namely the periphrastic construction such as "The block makes the toy go." However, the types of constructions languages use to depict causality are not limited to the periphrastic form (Comrie, 1989; Shibatani \& Pardeshi, 2002). Two other prevalent types of construction are lexical and morphological constructions. In lexical constructions, verbs encode both the cause and effect events. For example, in "Mary feeds the baby," the verb "feed" expresses both the feeding act of Mary as the cause and the eating of the baby as the effect. Morphological constructions mark the causal meaning by morphological modification such as attaching a causative morpheme to a verb stem. Some languages such as English express causatives with periphrastic or lexical constructions, whereas others also use morphological constructions. Turkish, for instance, uses both lexical constructions and morphological marking. Some causatives are expressed by lexical means using verbs such as "kes [cut]" and "kır [break]." Others are expressed by morphological constructions, whereby a causative morpheme is attached to a verb and turns it into a causative verb. For example, in the sentence "Mary bebeği ye-DIr-ir [Mary feeds the baby]," the causative morpheme "-DIr" is attached to "ye [eat]" to derive "yeDIr" [feed]. ${ }^{3}$

\footnotetext{
${ }^{3}$ The suffix can surface as one of “-dir, -dır, -dür, -dur, -tir, -tır, -tür, -tur" based on vowel harmony and consonant assimilation rules of Turkish.
} 
Each of these causative constructions can provide structural cues for a causal meaning. First, a shared feature of all these constructions is that they commonly have a transitive frame; that is, they involve two syntactic arguments (i.e., subject and object). Children have been shown to make use of this cue starting from the second year of life (e.g., Arunachalam \& Waxman, 2010; Fisher, 2002; Göksun, Küntay, \& Naigles, 2008; Kline, Snedeker, \& Schulz, 2017; Lidz, Gleitman, \& Gleitman, 2003; Naigles, 1990). For example, 2-year-olds choose a scene where a bunny pushes down a duck when they hear a pseudo-verb embedded in a transitive frame ("The bunny is gorping the duck") rather than a scene where they both wave one of their arms parallel to one another (Naigles, 1990). Second, the use of a distinct causality marker on the surface level is a feature that periphrastic and morphological causatives have in common. This feature is not shared with lexical causatives. That is, the predicate of cause in periphrastic constructions such as "make, let, cause" and the causative morpheme in morphological constructions such as "-DIr" in Turkish distinctly indicate a causal meaning. In contrast, lexical causatives such as "feed, break, push" embed the notion of causality within their semantics. Therefore, periphrastic and morphological causatives are structurally more transparent in expressing causality compared with lexical causatives (Shibatani \& Pardeshi, 2002).

The structural transparency of the causative constructions might influence how well children infer a causal meaning from what they hear. For example, if children do not yet know the meaning of a verb used in a sentence, they might more easily infer that it refers to a causal event when it involves a predicate of cause (e.g., Mary makes the baby gorp), or a causative morpheme (e.g., Turkish: Mary bebeği gorp-TUr-ur [Mary gorp-CAUS-PRES.3SG ${ }^{4}$ the baby]), than when it only appears in a transitive frame (e.g., Mary gorps the baby). These transparent surface-level cues may help children to perceive an event as causal that they might otherwise not perceive as causal.

In the current study, we focused on the understudied lexical and morphological constructions. For two reasons, we chose Turkish and Swiss German as target languages. First, Turkish employs both lexical and morphological causatives, whereas Swiss German employs only lexical but not morphological causatives. These systematic differences in the causative constructions in the two languages allowed us to compare the potential benefit of the more transparent morphological constructions with the less transparent lexical constructions on children's causal conceptualizations both within (Turkish) and across languages. Second, we aimed to extend the findings on the role of causal language in understanding untypical causal relations to languages other than English.

Two major research questions guided this research. First, does the structure of causal language alone (independent of verb meanings) facilitate children's understanding of untypical cause-effect relations? Second, do the different constructions of causality employed by different languages influence causal understanding to differing degrees?

With regard to the first question, there is ample evidence showing that children make use of the syntactic frame of sentences, as one structural cue, to derive causal meanings in several languages (e.g., Arunachalam \& Waxman, 2010; Fisher, 2002; Göksun et al., 2008; Kline et al., 2017; Lee \& Naigles, 2008; Lidz et al., 2003; Naigles, 1990). A recent study showed that 3- and 4-year-old children also make use of the morphological markers of causality to map onto causal events. Turkish-speaking children consistently mapped sentences involving a causative marker attached to a pseudo-verb onto causal events (Ger, Küntay, Goksun, Stoll, \& Daum, 2021). In studies that use a forced-choice design, where children choose one event from two alternatives upon hearing a sentence with structural cues of causality, the two alternatives are a typical causal and noncausal event (Arunachalam \& Waxman, 2010; Fisher, 2002; Ger, Küntay, et al., 2021; Kline et al., 2017; Naigles, 1990). In principle, children should already perceive these events as causal and noncausal and should choose the one fitting best with the sentence they hear. Therefore, we can only infer that children judge the sentence with a transitive frame or with a causative morpheme to better represent a causal event than a noncausal event. Previous research does not allow us to infer whether children represent an event that lacks typical causality features as causal only when it is described with a transitive frame or a causative morpheme. Therefore, we tested whether we can make children perceive a scene as causal when they hear it described with a causal construction using a pseudo-verb, which they might not otherwise readily

${ }^{4}$ gorp: a pseudo-verb; CAUS: causative morpheme; PRES: present tense; 3SG: third-person singular agreement. 
perceive as causal. If children did so, we could infer that the structure of the causal language alone can elicit a causal representation of an event.

With regard to the second question, morphological constructions are structurally more transparent than lexical constructions because they contain a distinct marker of causality on the surface level of a sentence. Therefore, we expected the Turkish-learning children to perceive causality easier when they hear a morphological construction depicting an untypical causal scene compared with when they hear a lexical construction. Moreover, with the same reasoning, we expected the Turkish-learning children who hear a morphological construction to perceive causality easier compared with the Swiss-Germanlearning children who hear a lexical construction. We did not expect a different performance between Turkish-learning and Swiss-German-learning children when they both hear a lexical construction or a noncausal construction.

We tested children at 2.5-3.5 years of age based on Bonawitz et al. (2010), who showed that at 4 years children were able to understand the untypical causal relations without the support of causal language, whereas at 2 years the causal language helped them. Hence, we predicted that children would benefit from causal language from 2 to 4 years of age. Given that we used pseudo-verbs in our design, which would remove crucial semantic information and presumably make the task even harder for 2-year-olds, we decided to set a slightly older minimum age.

To account for the possibility that children's causative comprehension might be influenced by their overall stage of language development, we assessed the vocabulary knowledge of the participants as a proxy for their linguistic knowledge. There is evidence for a robust correlation between children's grammar complexity and vocabulary size during the early stages of language development (Bates, Bretherton, \& Snyder, 1988; Dale, Dionne, Eley, \& Plomin, 2000; Hoff, Quinn, \& Giguere, 2018; Marchman \& Bates, 1994). Moreover, children with a larger vocabulary show a higher sensitivity of language-specific aspects of event representations (Göksun, Aktan-Erciyes, Hirsh-Pasek, \& Golinkoff, 2017).

In sum, first we asked whether syntactic and verbal morphological cues of causality in language facilitate children's causal understanding. We expected that in both Turkish and Swiss-German, children would understand cause-effect relations better in a causal language condition that employs these cues compared with a noncausal language condition. More specifically, we expected Turkish-learning children in both the lexical and morphological conditions to outperform those in the noncausal condition, and we expected Swiss-German-learning children in the lexical condition to outperform those in the noncausal condition. Second, we asked whether the different constructions of causality influence causal understanding to differing degrees. We expected Turkish-learning children in the morphological condition to outperform both Turkish- and Swiss-German-learning children in the lexical condition.

\section{Method}

\section{Participants}

The sample size was determined by an a priori power analysis for an analysis of covariance (ANCOVA) with five groups (for all language and condition groups) and one covariate (vocabulary score), a power of .90, an alpha level of .008 taking into account the correction for six planned comparisons $(.05 / 6)$, and a medium effect size $(r=.30)$ based on previous studies (Bonawitz et al., 2010; Butler \& Markman, 2012). This analysis determined a total sample size of 206 that pertained to 41.2 per group. Following this, we set our target sample size to 45 for each group. However, note that we later decided to use, and thus report, mixed-effects analyses, which are more powerful.

We tested 45 children in each condition, totaling 135 monolingual Turkish-learning children in three conditions and 90 monolingual Swiss-German-learning children in two conditions (see Table 1 for the descriptives of age and gender in each language and condition). A Kruskal-Wallis test showed that age did not differ across language and condition groups, $\chi^{2}(4)=4.20, p=.38$. An additional 32 Turkish-learning children and 2 Swiss-German-learning children were tested but excluded from the analyses due to a number of reasons (Turkish: 13 did not complete the task, 9 due to experimenter/ technical error, 4 were outside of target age, 4 had developmental disorders, 1 had a hearing problem, and 1 was bilingual; Swiss: 1 did not complete the task and 1 due to parent intrusion). 
Table 1

Descriptive statistics of age (in months) and gender in each language and condition group ( $n=45$ per group).

\begin{tabular}{|c|c|c|c|c|}
\hline & \multicolumn{2}{|l|}{ Turkish } & \multicolumn{2}{|l|}{ Swiss German } \\
\hline & Age & Gender & Age & Gender \\
\hline & $M(S D)$ [Range] & $n_{\text {female }}$ & $M(S D)$ [Range] & $n_{\text {female }}$ \\
\hline Noncausal & $35.9(4.3)[30-42]$ & 20 & $36.3(3.0)[30-41]$ & 29 \\
\hline Lexical & $37.1(3.7)[30-42]$ & 19 & $36.7(3.2)[30-42]$ & 21 \\
\hline Morphological & $37.4(3.4)[30-42]$ & 20 & - & - \\
\hline Overall & $36.8(3.9)[30-42]$ & 59 & 36.5 (3.1) [30-42] & 50 \\
\hline
\end{tabular}

Turkish-learning children were recruited from kindergartens of numerous districts of Istanbul, Turkey. Swiss-German-learning children were recruited from Zurich, Switzerland, via the participant database of the University of Zurich. All children received a small gift, and Swiss-German-learning children also received a symbolic certificate, for their participation. A demographic form was obtained from children's parents asking about parents' education level and whether children had siblings. Parents were also asked about whether children were exposed to another language in addition to the target language and, if so, the percentage of the exposure. Children were not tested if the exposure to (an) additional language(s) exceeded $15 \%$ of the total language input. ${ }^{5}$ The study was conducted in accordance with the Declaration of Helsinki and its later amendments. Informed consent was obtained from a parent for each child participant before the study.

Demographic information was obtained from $96 \%$ of the Swiss-German-learning participants and from $84 \%$ of the Turkish-learning participants. Based on these data, $72 \%$ and $47 \%$ of the SwissGerman- and Turkish-learning participants, respectively, had at least one sibling. For SwissGerman-learning children, $17 \%$ of mothers and $15 \%$ of fathers had completed a secondary education (high school or vocational training equivalent), and $82 \%$ of mothers and $84 \%$ of fathers had completed a tertiary education (university equivalent). For Turkish-learning children, $9 \%$ of mothers and $16 \%$ of fathers had completed 5 or 8 years of compulsory education (primary school equivalent), 35\% of mothers and $29 \%$ of fathers had completed a secondary education (high school or vocational training equivalent), $43 \%$ of mothers and $50 \%$ of fathers had completed a tertiary education (university equivalent), and $12 \%$ of mothers and $5 \%$ of fathers had completed a higher education (Master's or Ph.D. degree).

\section{Materials and design}

We used a task adapted from Bonawitz et al. (2010). The task consisted of training and four test trials, with each trial being composed of an observation phase and an intervention phase. Fig. 1 shows all the materials used in the intervention phase. These consisted of four wooden "cause objects," four battery-powered and mobile "effect objects," four immobile "distractor objects," two white boxes with an opening in the back where cause objects and distractor objects were stored, and switches to turn the effect objects on and off. The effect objects were not physically connected to the cause objects and were operated by the experimenter. They were attached to switches with cables and were mounted on wooden plates underneath and behind the objects. The cables went through little holes on the plate behind the objects to make them invisible to the children. The switches were also hidden under the table to let the experimenter operate them surreptitiously. Preceding the test trials, training was included to familiarize children with the task of first watching a video and then, in real life, acting on the objects they had watched in the video. For the training, stacking cups placed on white cardboard and a video of a female actor stacking the cups were used.

The materials used in the observation phase of the trials consisted of eight videos (two sets of four videos) that were presented on a 17-inch touchscreen laptop computer using the software PsychoPy Version 1.90.1 (Peirce \& MacAskill, 2018). The videos and the Python script for the experiment can be found in this link: materials. The videos contained the described cause and effect objects as well as

\footnotetext{
${ }^{5}$ Note that 1 bilingual Turkish-learning child was tested by mistake and was excluded from the analyses.
} 


Lause objects
$\begin{aligned} & \text { Lever can be slid to the left, when } \\ & \text { released flips back to original } \\ & \text { position }\end{aligned}$
$\begin{aligned} & \text { Sisk can be lifted upwards, when } \\ & \text { released falls back down }\end{aligned}$
$\begin{aligned} & \text { Pendulum } \\ & \text { small wooden plate on the right } \\ & \text { side; when released returns to } \\ & \text { central position } \\ & \text { Button box } \\ & \text { Button can be pushed inwards, } \\ & \text { when released comes back out }\end{aligned}$

Fig. 1. Materials used in the intervention phase (For the color version of this figure, see the Web version of this article).

four "agent objects" that were wooden blocks in shapes of a cube, a cylinder, a sphere, and a pyramid. Each agent object was paired with a fixed cause object (cube with pendulum, cylinder with lever box, sphere with button box, and pyramid with ramp). Each cause object was paired with either of two effect objects: one that has an effect in two modalities (such as movement and light) and one that has an effect in only one modality (such as only light). The videos were grouped into two sets of four videos, so that in each set all cause objects and all effect objects were used once (Set A: lever box + house, ramp + bird, pendulum + car, button box + flower; Set B: lever box + bird, ramp + house, pendulum + flower, button box + car). There were four presentation orders for each set in a Latin square design. Children were randomly assigned to a set and a presentation order.

For the vocabulary assessment, the BILEX scale (Gampe, Kurthen, \& Daum, 2018) was used. This scale was developed to assess bilingual children's lexicon and translational equivalents (whether children know a word in both of their languages) by following the logic of the Peabody Picture Vocabulary Test (Dunn, 2019). Therefore, the same set of pictures for target items was labeled and recorded by native speakers of several different languages. The BILEX scale is available in both target languages used in the current study. Therefore, it was possible to assess the vocabulary of children speaking Turkish or Swiss German with the same items. The task was administered on a touchscreen laptop. Children were presented with the spoken label of one object and, at the same time, saw an arrangement of six pictures on the touchscreen. Children were asked to tap on the corresponding picture of the word they heard. The stimuli consisted of a total of 48 pictures composed of animals, buildings, furniture, fruits, and vegetables (for the materials, see https://osf.io/x5wcj/).

In the experimental task, children were tested in the following experimental conditions: Turkish: noncausal, lexical, and morphological; Swiss German: noncausal and lexical. The videos of the observation phase had an embedded auditory description recorded by a native female speaker that varied based on the condition (e.g., noncausal: Here is a cube and a car; lexical: The cube is gorping the car; morphological: The cube is gorp-CAUS-ing ${ }^{6}$ the car). In the intervention phase following the observation,

${ }^{6}$ CAUS indicates the causative morpheme in Turkish that is suffixed to a pseudo-verb. 
a prompt corresponding to the condition was used (e.g., noncausal: Now you can play; lexical: Now you can gorp the car; morphological: Now you can gorp-CAUS the car). A different pseudo-verb was used in each of the description and prompt pairs. See Table 2 for the descriptions and following prompts in each language and condition.

\section{Procedure}

The Swiss-German-learning children were tested in the lab at the University of Zurich. Parents and children were first welcomed in a play room where children were familiarized with the experimenter. Then they proceeded to a testing room with the experimenter. Children sat at a table next to the experimenter on a chair, and parents sat behind the children. The Turkish-learning children were tested in a quiet room in kindergartens around Istanbul. First, the experimenter was introduced to the children in a playroom in the kindergarten, and children were then taken to a quiet room individually for the testing. Children sat at a table across from the experimenter on a chair. There was a warm-up phase where the experimenter chatted with children for a few minutes before starting testing. Children and the experimenter were alone in the room throughout the testing. At both sites, children were videotaped by two cameras (at the front and back of children at the Turkish site; at the front of children and from the ceiling at the Swiss site). Testing was conducted at the two sites by native speakers of the corresponding languages.

First, children were instructed that they were going to see a series of videos and that after each video they were supposed to do something. Then the training was done to familiarize children with the task of first watching a video and then, in real life, acting on the objects they saw in the video. In the training video, which was played twice, four stacking cups stood next to each other in an order from the smallest to the largest, and a female actor stacked the four stacking cups on top of each other in the correct order. Then the experimenter placed the same stacking cups in front of children on cardboard in the same order and prompted children as follows: "Now it is your turn. Now you can do it." If children did not act on the cups, the experimenter prompted them again verbally, and if the second verbal prompt did not work, the experimenter prompted them further by stacking the first cup. Children were given 1-2 min to act on the cups. When children finished stacking the cups, regardless of whether it was the correct order or not, the experimenter took the cups away while saying "You played really well" and moved on to the test trials.

In the observation phase of each trial, children saw a video that lasted approximately $10 \mathrm{~s}$. In this video, an agent object (e.g., a wooden cube) moved and acted on a cause object (e.g., pushing the button on the button box). At the time of the act on the cause object, an effect object that stood at a distance turned on (e.g., a flower-shaped mini-fan lighted up and started spinning) and stopped as soon as the agent object moved away from the cause object (e.g., let go of the button). Hence, neither the agent object nor the cause object physically contacted with the effect object. The video had an auditory description based on the experimental condition and language group (e.g., noncausal: Here is a cube and a flower; lexical: The cube is gorping the flower). The video presentation was repeated six times, and then the laptop was taken away.

In the intervention phase, the experimenter placed the same effect object that the children had seen in the video on top of a box that stood on one end of the table (i.e., to the left or right of the child) while saying "I put this here." In addition, she placed a distractor object (e.g., a rattle) on top of a second box that stood on the opposite end of the table while saying "And this I put here." Finally, she held the same cause object that the children had seen in the video in front of the children while saying "And I have something for you-here it is." Then the experimenter prompted the children based on the condition to which children were assigned (e.g., noncausal: Now you can play; lexical: Now you can gorp the flower). This was done once while the experimenter was still holding the object and once again while she placed it in front of the children on the table. The experimenter then sat next to the children (across from the children at the Turkish site) and secretly placed her hand on the switches. If the children performed the target action on the cause object, the experimenter waited a few seconds and surreptitiously turned on the effect object for 3-4 s. This brief waiting period was used to detect the children's expectant looks to the effect object. The effect object was activated by the experimenter 


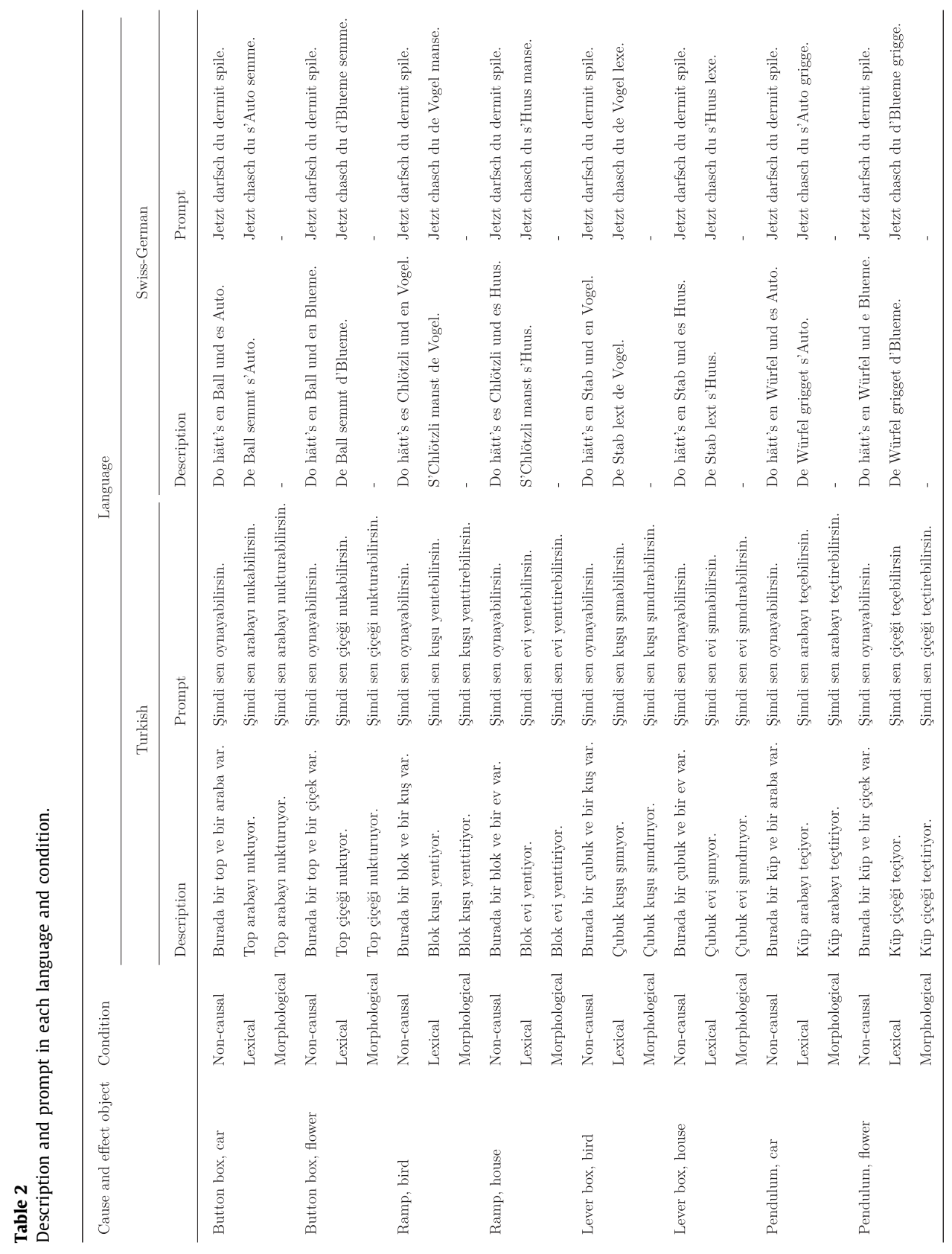


a maximum of two times. The experimenter then continued to the next trial while saying "You played really well. Now you will watch a different video."

Two of the distractor objects and two of the effect objects were hidden inside one of the boxes, and the other two of each were hidden inside the other box. The experimenter always started placing the object on the box to her right, resulting in the distractor object being placed first in half of the trials and the effect object being placed first in the other half.

\section{Coding and scoring}

We used the software ELAN (Sloetjes \& Wittenburg, 2008) to annotate target behaviors of children and activation of effect objects frame by frame, time-aligned to the video recordings of the testing sessions. For the intervention phase, we coded whether the children performed the target action on the cause object for each trial. The target actions were (a) pushing the button on the button box, (b) sliding the lever on the lever box, (c) lifting the knob upward on the ramp, and (d) pushing the pendulum toward the base. We considered children to have performed the target action even when they did not push the button, lever, or knob all the way but did so noticeably considering that they might have had physical difficulties in doing so (except in the case of the pendulum, where children were expected to make it touch the base because it was easy enough to move). We also coded whether the children looked to the effect object or the distractor object while and/or after performing the target action and annotated the duration of the looks. Because we were only interested in the predictive looks, we did not code any looks that occurred before performing the target action.

For each trial, performance was considered successful and given a score of 1 if children looked at the effect object for a duration of at least $500 \mathrm{~ms}$ within $2 \mathrm{~s}$ following the start of performing the target action. In contrast to Bonawitz et al. (2010), we did not assess whether children learned the predictive relation between the agent object's contact with the cause object and the activation of the effect object. The reasons for this were as follows. First, Bonawitz and colleagues showed that nearly all toddlers learned this predictive relation. Second, Muentener et al., 2012 showed that causal language helped toddlers already in representing the predictive relations as causal rather than only helping them in initiating causal interventions. Therefore, we assumed that children's failure would be due to failing to represent the predictive relations as causal and hence potentially reproducible. Performance was considered unsuccessful and given a score of 0 if (a) children did not perform the target action (no action), (b) children did not look to either of the objects for at least $500 \mathrm{~ms}$ during or after performing the target action (no look), (c) children's first look of at least 500 ms was directed to the distractor object but later than $2 \mathrm{~s}$ following the start of performing the target action (late look to distractor), (d) children's first look of at least $500 \mathrm{~ms}$ within $2 \mathrm{~s}$ following the start of performing the target action was directed to the distractor object (expectant look to distractor), or (e) children's first look of at least $500 \mathrm{~ms}$ was directed to the effect object but later than $2 \mathrm{~s}$ following the start of performing the target action (late look to effect object). The total number of successful trials ranged from 0 to 4 . The first author, who was not blind to condition, coded half of the Turkish-learning participants' data and $10 \%$ of the Swiss-German-learning participants' data. The second author, who was not blind to condition, coded half of the Swiss-German-learning participants' data. The first and second authors had $100 \%$ agreement. A third naive coder, who was blind to condition, coded the remaining half of the participants in each language group. The blind coder and the first author ensured high reliability $\left(r_{\mathrm{s}}=.97, \mathrm{kappa}=.91\right)$ from $10 \%$ of the jointly coded Swiss-German data and $15 \%$ of the jointly coded Turkish data.

For the training, we also coded (1) "inactive" when children did not act on the cups at all, (2) "active" when children stacked some or all of the cups but not in the correct order, or (3) "correct" when children stacked all the cups in the correct order. The training was implemented to increase children's motivation to act on the cause objects in the test trials and was not intended to be used as an inclusion criterion. Overall, only 4 children were inactive in the training, and 2 of them performed the target action in all four trials, whereas the remaining 2 performed the target action in at least two of the four trials. Therefore, we did not exclude any children from the final analyses based on inactivity during training. However, we still coded the type of performance in three categories to explore whether this performance had an influence on the success in test trials. 


\section{Results}

We used R Version 4.0.2 (R Core Team, 2020) for all analyses. The data and analysis script can be found in this link: data \& analyis script

We conducted a series of mixed-effects models to examine the effect of language group and condition, controlling for age, on the likelihood of successfully completing a trial. In each model, we entered participant as a random effect to account for the variance caused by multiple measurements from the same individuals. First, we checked whether children's sex, the performance in the training, the video set or order, and the trial number had an influence on children's likelihood of success in the test trials. To do so, we compared each of the five mixed models with participant as the random effect and sex, training performance, video set, order, or trial number as the fixed effect with a model with only intercept and participant as the random effect. The model comparisons for all but the trial number yielded nonsignificant chi-square values, indicating that none of these factors except the trial number influenced the test performance [sex: $\chi^{2}(1)=2.80, p=.09$; training performance: $\chi^{2}(2)=2.80, p=.25$; video set: $\chi^{2}(1)=0.45, p=.50$; order: $\chi^{2}(7)=9.50, p=.22$; trial number: $\left.\chi^{2}(3)=112.00, p<.0001\right]$. Therefore, we included only the trial number in the final model.

Because the Swiss-German group lacked the morphological condition, the parameter estimates with the language and condition would result in a missing cell. Therefore, we defined a new variable of language condition [with five levels: (a) Swiss-German noncausal, (b) Swiss-German lexical, (c) Turkish noncausal, (d) Turkish lexical, and (e) Turkish morphological] and treated all five groups independently in examining the influence of the language group and condition. We also checked whether the vocabulary score of children influenced test performance particularly in the causal conditions to account for the possibility that children with larger vocabularies show higher sensitivities to language-specific cues in event representations (Göksun et al., 2017). To do so, we first checked whether children's vocabulary scores were comparable across the language and condition groups. A linear regression model predicting vocabulary score from language, condition, and their interaction revealed a significant effect of language, $F(1,215),=48.50, p<.0001$, but a nonsignificant interaction, $F(1,215)=0.42, p=.52^{7}$. Overall, Swiss-German-learning children $(M=38.10, S D=5.55$, range $=25-47)$ scored higher than Turkish-learning children $(M=32.30, S D=5.72$, range $=14-43)$. Therefore, we centered and scaled the vocabulary scores based on the language groups and ran a mixed model with language condition, vocabulary score, and their interaction as fixed effects and participant as the random effect. An analysis of variance (ANOVA) on the model yielded a significant effect of vocabulary score $\left(\chi^{2}(1)=11.14, p<.001\right)$ but a nonsignificant interaction $\left(\chi^{2}(4)=0.80, p=.94\right)$. This indicated that vocabulary size had an overall influence on children's performance regardless of condition and hence no particular influence on their ability to make use of linguistic causality cues.

Consequently, we ran the final model by using the following lme4 syntax: success $\sim$ language condition + age + trial number $+(1 \mid$ participant ID). The age was a significant predictor (estimate $=0.10$, $S E=0.03, z=3.32, p<.001$ ), such that a 1 -month increase in age was associated with a 1.11 times increase in the log of odds of successfully completing a trial. Note that adding an interaction term between language condition and age to the model did not yield a significant improvement in the model fit, indicating no significant interaction. This additional analysis can be found in the online supplementary material. The trial number was also a significant predictor (estimate $=0.77, S E=0.08$, $z=9.45, p<.0001$ ), indicating a learning effect across trials. More specifically, the log of odds of successfully completing a trial increased by 2.16 times with each subsequent trial. Fig. 2 shows the predicted probability of success across age and trial number.

To examine whether this learning effect (over the trials) differed as a function of language condition, we compared the final model (by dropping the age variable to circumvent convergence problems) with a model with an interaction term of language condition and trial number. The interaction term did not provide a significant contribution to the model, $\chi^{2}(4)=4.51, p=.342$, indicating that there was a similar slope of learning across the different language groups and conditions.

\footnotetext{
${ }^{7}$ Note that there were 5 missing values ( 3 Swiss German and 2 Turkish) as a result of not completing the vocabulary assessment due to fussiness; however their total number of successful trials did not significantly differ from the rest $(p=.45)$.
} 

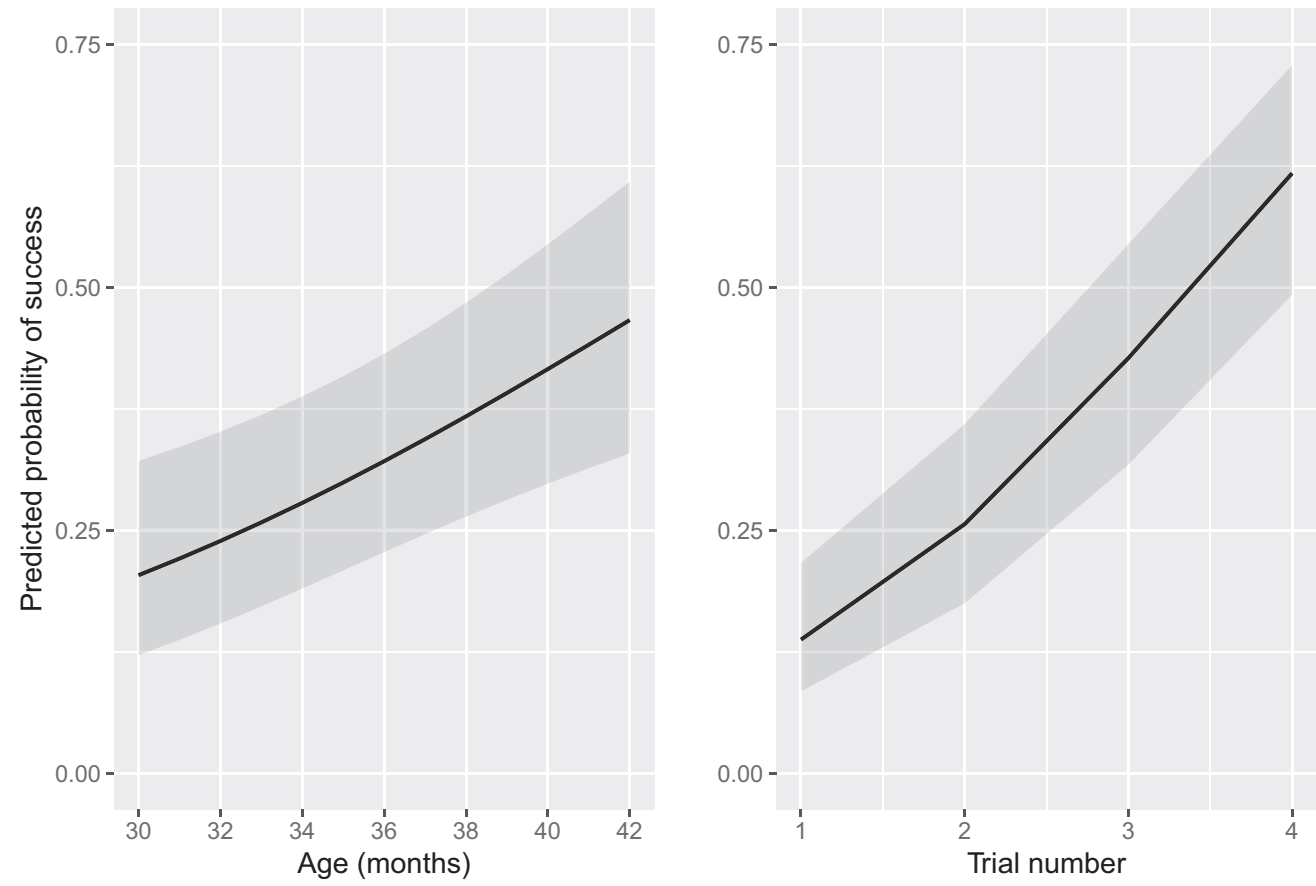

Fig. 2. Predicted probability of success across age (left) and trial number (right).

To further investigate the language and condition effects in the final model, we ran six planned contrast analyses using the emmeans function with a custom contrast matrix and using the mvt method for $p$-value adjustment (Lenth, 2020). Two of these contrasts concerned the first question of whether the structure of causal language facilitates causal representation. These contrasts were (1) Swiss-German noncausal compared with Swiss-German lexical and (2) Turkish noncausal compared with the average of Turkish lexical and Turkish morphological. The remaining four contrasts concerned the second question of whether the different constructions of causality employed by different languages influence causal understanding to differing degrees. These contrasts were (3) Turkish morphological compared with Turkish lexical, (4) Turkish morphological compared with SwissGerman lexical, (5) Turkish lexical compared with Swiss-German lexical, and (6) Turkish noncausal compared with Swiss-German noncausal. Table 3 shows the results of these contrast analyses. There were significant differences only between Swiss-German noncausal and Swiss-German lexical and between Turkish noncausal and the average of Turkish lexical and Turkish morphological groups. The log of odds of succeeding in a trial was 2.8 times more likely for Swiss-German-learning children in the causal language condition than in the noncausal condition and was 2.3 times more likely for Turkish-learning children in a causal language condition than in the noncausal condition. The Turkish morphological group was not more likely to succeed in a trial than the lexical group in either of the languages. Fig. 3 shows the predicted probability of successfully completing a trial for each language and condition.

We further explored the unsuccessful trials to demonstrate the possible range of responses and how they were distributed across the language groups, conditions, and trials. Fig. 4 shows the number of children at different performance levels across trials. To be able to compare the number of children falling into each performance category across the different conditions with a chi-square test, we grouped children's performance into three categories to have a sufficient number of participants in each cell. We combined the "no action," "no look," and "late look to distractor" outcomes into a "poor performance" category, combined the "expectant look to distractor" and "late look to effect" outcomes 
Table 3

Results of planned contrast analyses.

\begin{tabular}{llllll}
\hline Contrasts & Estimate & exp(Est) & $S E$ & $z$ Ratio & $p$ Value \\
\hline 1. SG lexical-SG noncausal & 1.02 & 2.8 & 0.34 & 2.98 & .016 \\
2. T lex-morph-T noncausal & 0.83 & 2.3 & 0.30 & 2.80 & .028 \\
3. T morph-T lexical & 0.06 & 1.1 & 0.34 & 0.19 & .999 \\
4. T morph-SG lexical & 0.18 & 1.2 & 0.34 & 0.53 & .977 \\
5. T lexical-SG lexical & 0.12 & 1.1 & 0.34 & 0.35 & .996 \\
6. T noncausal-SG noncausal & 0.34 & 1.4 & 0.34 & 0.99 & .814 \\
\hline
\end{tabular}

Note. exp(Est), exponential of the estimate; T, Turkish; SG, Swiss-German; lex-morph, average of the lexical and morphological conditions.

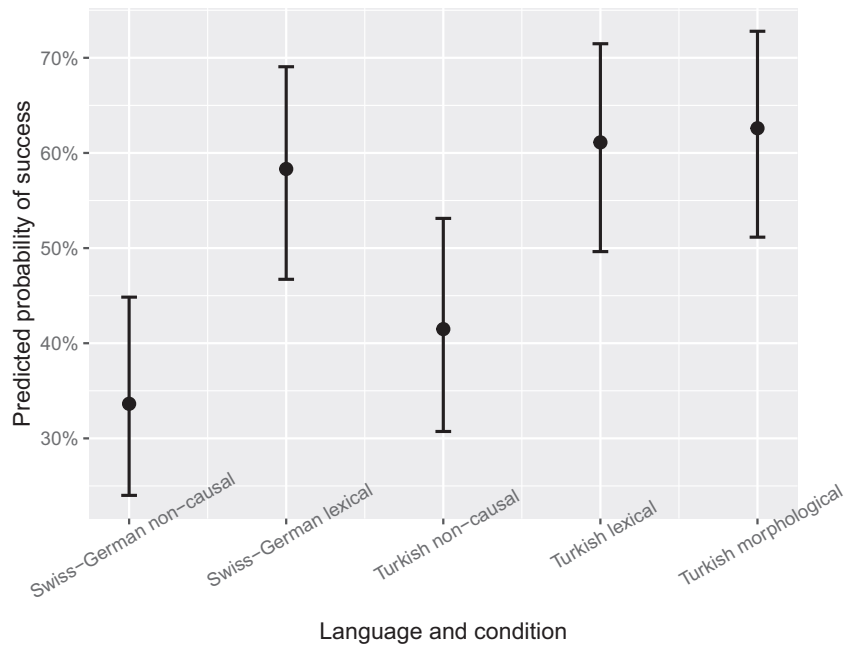

Fig. 3. Predicted probability of successfully completing a trial in each language and condition. Error bars represent $95 \%$ confidence intervals.

into a "moderate performance" category, and kept the "success" category. We looked at the noncausal and causal groups separately and for each trial separately. For the noncausal conditions, there was no significant effect of language group on the performance in any of the trials (all $p s>.05$ ). For the causal conditions, there was a significant effect of language condition only for the first trial, $\chi^{2}(4)=10.37$, $p=.03$, Cohen's $d=0.58$. A post hoc test with Bonferroni correction showed that Swiss-Germanlearning children (in the lexical condition) were more likely to show "poor performance" than Turkish-learning children in either of the conditions $(p=.02)$.

\section{Discussion}

In the current study, we addressed the following two research questions. First, we tested whether the structural cues of causal language facilitate young children's understanding of untypical causal events. We found that the lexical causal constructions in both Turkish and Swiss German, as well as the morphological causal construction in Turkish, led children to understand causality better than a noncausal construction. This suggests that the structural cues of causal language facilitate children's causal understanding. We found no difference between the performance of the noncausal groups in Turkish and Swiss German. This suggests that children in both language groups understand the cause-effect relations in the videos to similar extents without the support of linguistic cues. Second, we tested whether the different cues as exhibited in Swiss-German and Turkish languages have a dif- 


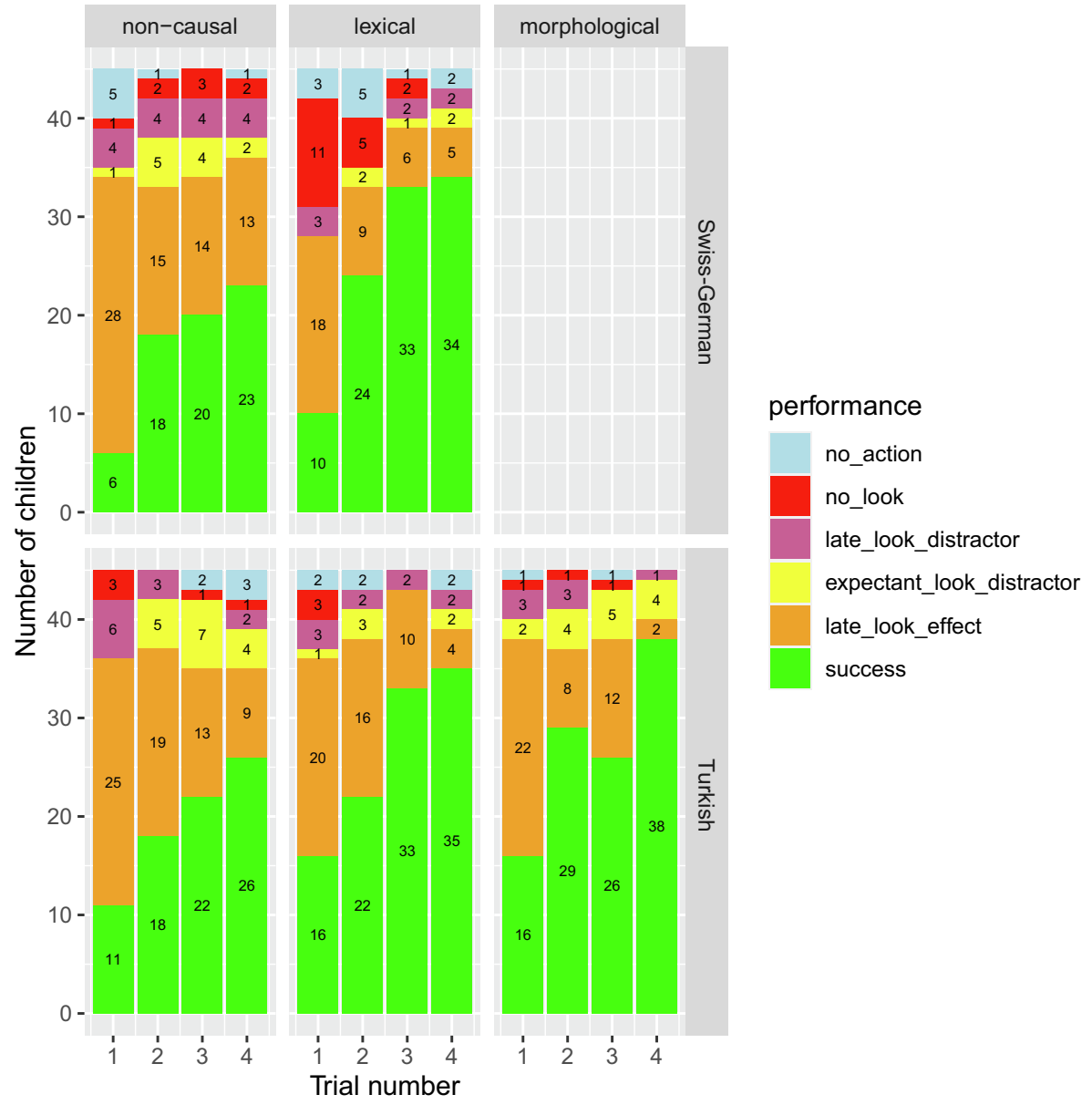

Fig. 4. Numbers of children with different performances across language groups, conditions and trials (For the color version of this figure, see the Web version of this article).

ferent effect on children's causal understanding. We found no difference between the types of causal construction either within Turkish or across these two language groups. In particular, in line with our expectation, performance of Turkish-learning children in the lexical condition did not differ from that of Swiss-German-learning children in the lexical condition. However, contrary to our expectation, performance of Turkish-learning children in the morphological condition also did not differ from that of both language groups in the lexical condition. Together, the findings suggest that at 2.5-3.5 years of age, causal language constructions facilitate children's causal inferences, but the type of construction does not influence the extent of this facilitation.

We provided corroborating evidence that children at toddler to preschool ages benefit from adults' causal descriptions to represent events as causally connected when the events lack the typical contact or dispositional agency cues of causality (cf. Bonawitz et al., 2010; Butler \& Markman, 2012). We contributed to the existing literature by showing that this benefit holds true for speakers of two typologically different languages, other than English. More important, we provided evidence that the structural cues of causal language are sufficient to bring about this benefit. That is, children speaking Turkish or Swiss German conjectured a causal meaning even from an unknown (pseudo) verb as long as it was used in a sentence that contained structural cues to causality (i.e., syntactic frame or syntac- 
tic frame and verbal morphological cues). They then used this information to reconceptualize the presented events as causally related.

The findings also extend the literature regarding the causal form-meaning mapping (Arunachalam \& Waxman, 2010; Kline et al., 2017; Naigles, 1990). Children not only infer a causal meaning from these linguistic cues but also use these cues to modify their initial noncausal event representations to include potential causal links between events. Children perhaps represent the cause event (e.g., the cube pushing the button box) and the effect event (e.g., the mini fan spinning) in our task as two unrelated events in the absence of causal language and represent them as causally related subevents only when the accompanying language contains cues that signal a causal meaning. This is in line with the idea that language provides children with access to concepts that are otherwise not readily accessible (Nelson, 2005). Research also shows that when objects are described by causal features (e.g., "This is a tib [a square object]. It makes the machine go"), toddlers and preschoolers categorize them based on these causal features, such as grouping all objects that made the machine work as tibs, rather than on perceptual features, such as grouping all square objects as tibs (Gopnik \& Sobel, 2000; Nazzi \& Gopnik, 2000).

Children were not able to make use of linguistic causality cues right away but rather needed some practice until they could reliably use these cues to restructure their initial, presumably noncausal representations of the observed scenes into causally connected representations. Although both language groups in all conditions (including noncausal language) showed similar learning over the trials, overall children in the causal language conditions outperformed those in the noncausal language conditions. This suggests that causal descriptions in any construction type and in both languages were effective in supporting children's causal conceptualizations. However, unsurprisingly, without the semantics of the verbs, children seem to have a harder time incorporating the causal language cues into their event representations (cf. Bonawitz et al., 2010; Butler \& Markman, 2012).

We expected that the morphological construction of causality, with its surface-level transparency and certainty in expressing a causal meaning, would provide an exclusive benefit for children to represent seemingly unrelated events as causally connected. However, we did not find such an advantage of morphological causatives over lexical causatives, at least not in the age period we tested. A reason why the morphological causatives did not lead to a strongly enhanced causal representation could be that the Turkish children might still be developing their morphological analyses and segmentation abilities at 2.5-3.5 years of age. Relatedly, some children in this condition might have perceived the causatively marked pseudo-verbs as unanalyzed chunks. Perhaps it needs more linguistic experience until children can reliably detect the causative marker and analyze it as a meaningful unit denoting causality. In fact, a recent study using pseudo-verbs found that Turkish children could not reliably judge the causative marker to be used for the causativized version of an event until the end of 4 years of age (Ger, You, et al., 2021). Moreover, despite improving from second grade to fourth grade in school, Turkish-speaking children still made errors in correcting incorrectly used inflections or providing correct inflections on pseudo-verbs (Durgunoğlu, 2003). Furthermore, even in morphologically less rich languages such as English, the productive use of inflections on pseudo-verbs continues to develop into school age (Berko, 1958). Therefore, the possibility remains that some Turkish children in the current study still lacked the rule-based understanding of the causative marker "-DIr" that hindered them from making use of it to represent the causality in events. It would be interesting to see in future studies whether an advantage of morphological causatives on children's causal inferences emerges at a later age in development.

Children's causal conceptualization of the events increased with age independent of the language and condition groups. It is an expected pattern that children incorporate a wider variety of causal relations into their repertoires with increasing age. Whereas younger children are mostly bound to the typical features of causality such as the agency of causers and the physical contact between the agent and patient (e.g., Kotovsky \& Baillargeon, 2000; Muentener \& Carey, 2010), older children go beyond these constraints even without the additional support of causal language (e.g., Meltzoff et al., 2012). Hence, older children in our study might have been more flexible in interpreting a causal relation by accepting inanimate agents and distal outcomes. As children grow older, they are also increasingly adept at updating their prior beliefs based on incoming statistical evidence regarding potential causal links (Koerber, Sodian, Thoermer, \& Nett, 2005; Schulz, Bonawitz, \& Griffiths, 2007). Children in our 
study were presented with the videos a number of times where they saw the causal evidence. Therefore, older children, even if they had prior beliefs that goal-directed agency and physical contact are essential in causal relations, might have updated these beliefs with the repeated evidence better than younger children.

In the exploratory analyses of the different performance levels, we found a difference only in the first trial of the total four trials. Specifically, the Swiss-German-learning children in the lexical condition were more likely to show "poor performance" than the Turkish-learning children in either of the causal conditions. No difference emerged for the noncausal conditions. This might suggest that children who grow up with a language that includes both syntactic and morphological cues of causality in everyday speech could have an advantage in "quickly perceiving" causal connections (given that the effect was present only in the first trial) compared with children who grow up with a language that includes only syntactic cues. Nonetheless, one limitation of our study is that in both the morphological and lexical conditions in Turkish, an additional nominal case marking cue (i.e., accusative marked object) was present, which Swiss German does not employ. Turkish-learning children aged 2-5 years have been shown to make use of the accusative case marker in their causal interpretations (Göksun et al., 2008). Therefore, these case markers might have facilitated Turkish-learning children's causal event representations in the causal conditions. However, given that Turkish-learning children in either of the two conditions were no more likely to successfully complete a trial than the SwissGerman-learning children who did not have these case markers but needed to rely solely on the transitivity cue, the nominal case marker in Turkish is not likely to be the main cue that children focused on for their causal interpretations.

A second limitation of the current study is that the sentences in the morphological condition in Turkish had both a transitive syntactic frame and the verbal causative marker. If, as hypothesized, children in this condition had outperformed children in the lexical condition in Turkish, we could have inferred that the verbal morphological cue had provided an additional benefit in causal representations. However, given that there was no difference in performance between these two groups, we cannot confidently infer that the facilitation of causal comprehension in the morphological condition was merely due to the morphological cue. Children might have paid attention only to the transitive frame without having perceived the causative marker attached to the pseudo-verb. A future direction would be to more reliably disentangle the influence of the transitivity and the causative marker cues in Turkish.

To conclude, this study provides further evidence that young children benefit from causal language to extend their causal representations to inanimate agents and distal effects (where there is no direct physical contact between the cause and the effect). Crucially, this benefit holds even without the use of familiar verbs with causal meanings but with only structural causality cues in the language, namely the transitive syntactic frame and the verbal morphological causative marker. From toddler to preschool ages, children might use their linguistic representations of causality, which might initially be constrained to typical causal relations, to incorporate new causal agents and causal relations. Typological differences between Turkish and Swiss German in how causality is represented do not seem to influence young learners' cognitive representations of causality. Nevertheless, the causative morpheme in Turkish, which is a more transparent and reliable indicator of causality, might provide additional support for causal event representations at an older age when children have more robust skills in morphological analyses that can incorporate novel verbs and contexts. Thus, crosslinguistic differences in the domain of causal cognition ought to be further explored.

\section{Declaration of Competing Interest}

The authors declare that they have no known competing financial interests or personal relationships that could have appeared to influence the work reported in this paper.

\section{Appendix A. Supplementary material}

Supplementary data to this article can be found online at https://doi.org/10.1016/j.jecp.2021. 105182. 


\section{References}

Arunachalam, S., \& Waxman, S. R. (2010). Meaning from syntax: Evidence from 2-year-olds. Cognition, 114, 442-446. https://doi. org/10.1016/j.cognition.2009.10.015.

Bates, E., Bretherton, I., \& Snyder, L. (1988). Acquisition of a novel concept at 20 months. In From first words to grammar: Individual differences and dissociable mechanisms (pp. 124-134). Cambridge, UK: Cambridge University Press..

Berko, J. (1958). The child's learning of English morphology. Word, 14, 150-177. https://doi.org/10.1080/ 00437956.1958.11659661.

Bonawitz, E. B., Ferranti, D., Saxe, R., Gopnik, A., Meltzoff, A. N., Woodward, J., \& Schulz, L. E. (2010). Just do it? Investigating the gap between prediction and action in toddlers' causal inferences. Cognition, 115, 104-117. https://doi.org/10.1016/j. cognition.2009.12.001.

Butler, L. P., \& Markman, E. M. (2012). Finding the cause: Verbal framing helps children extract causal evidence embedded in a complex scene. Journal of Cognition and Development, 13, 38-66. https://doi.org/10.1080/15248372.2011.567201.

Carey, S., \& Spelke, E. (1994). Domain-specific knowledge and conceptual change. In L. A. Hirschfeld \& S. A. Gelman (Eds.), Mapping the mind: Domain specificity in cognition and culture (pp. 169-200). New York: Cambridge University Press..

Cohen, L. B., \& Amsel, G. (1998). Precursors to infants' perception of the causality of a simple event. Infant Behavior and Development, 21, 713-731. https://doi.org/10.1016/S0163-6383(98)90040-6.

Comrie, B. (1989). Language universals and linguistic typology: Syntax and morphology. Chicago: University of Chicago Press. Dunn, D. (2019). Peabody picture vocabulary test (5th ed.). Bloomington, MN: Pearson..

Dale, P. S., Dionne, G., Eley, T. C., \& Plomin, R. (2000). Lexical and grammatical development: A behavioural genetic perspective. Journal of Child Language, 27, 619-642. https://doi.org/10.1017/S0305000900004281.

Dunn, D. (2019). Peabody picture vocabulary test (5th ed.). Bloomington, MN: Pearson..

Durgunoğlu, A. Y. (2003). Recognizing morphologically complex words in Turkish. In E. M. H. Assink \& D. Sandra (Eds.), Reading complex words: Cross-language studies (pp. 81-92). Boston: Springer. https://doi.org/10.1007/978-1-4757-3720-2_4.

Fisher, C. (2002). Structural limits on verb mapping: The role of abstract structure in 2.5-year-olds' interpretations of novel verbs. Developmental Science, 5, 55-64. https://doi.org/10.1111/1467-7687.00209.

Gampe, A., Kurthen, I., \& Daum, M. M. (2018). BILEX: A new tool measuring bilingual children's lexicons and translational equivalents. First Language, 38, 263-283. https://doi.org/10.1177/0142723717736450.

Ger, E., Küntay, A. C., Goksun, T., Stoll, S., \& Daum, M. M. (2021, May 31). How do different language typologies influence causal event conceptualizations? https://doi.org/10.31234/osf.io/p3dgc.

Ger, E., You, G. Küntay, A. C., Göksun, T., Stoll, S., \& Daum, M. M. (2021). Late generalization of morphological causatives: Evidence from Turkish. Cognitive Science. Submitted for publication..

Göksun, T., Aktan-Erciyes, A., Hirsh-Pasek, K., \& Golinkoff, R. M. (2017). Event perception and language learning. In F. N. Ketrez, A. C. Küntay, Ş. Özçalışkan, \& A. Özyürek (Eds.), Social environment and cognition in language development: Studies in honor of Ayhan Aksu-Koç (pp. 179-198). Philadelphia: John Benjamins. https://doi.org/10.1075/tilar.21.12gok.

Göksun, T., Küntay, A. C., \& Naigles, L. R. (2008). Turkish children use morphosyntactic bootstrapping in interpreting verb meaning. Journal of Child Language, 35, 291-323. https://doi.org/10.1017/S0305000907008471.

Gopnik, A., Glymour, C., Sobel, D. M., Schulz, L. E., Kushnir, T., \& Danks, D. (2004). A theory of causal learning in children: Causal maps and Bayes nets. Psychological Review, 111, 3-32. https://doi.org/10.1037/0033-295X.111.1.3.

Gopnik, A., \& Sobel, D. M. (2000). Detecting blickets: How young children use information about novel causal powers in categorization and induction. Child Development, 71, 1205-1222. https://doi.org/10.1111/1467-8624.00224.

Hoff, E., Quinn, J. M., \& Giguere, D. (2018). What explains the correlation between growth in vocabulary and grammar? New evidence from latent change score analyses of simultaneous bilingual development. Developmental Science, 21, e12536. https://doi.org/10.1111/desc.12536.

Kline, M., Snedeker, J., \& Schulz, L. (2017). Linking language and events: Spatiotemporal cues drive children's expectations about the meanings of novel transitive verbs. Language Learning and Development, 13, 1-23. https://doi.org/10.1080/ 15475441.2016.1171771.

Koerber, S., Sodian, B., Thoermer, C., \& Nett, U. (2005). Scientific reasoning in young children: Preschoolers' ability to evaluate covariation evidence. Swiss Journal of Psychology, 64, 141-152. https://doi.org/10.1024/1421-0185.64.3.141.

Kotovsky, L., \& Baillargeon, R. (2000). Reasoning about collisions involving inert objects in 7.5-month-old infants. Developmental Science, 3, 344-359. https://doi.org/10.1111/1467-7687.00129.

Lee, J. N., \& Naigles, L. R. (2008). Mandarin learners use syntactic bootstrapping in verb acquisition. Cognition, 106, $1028-1037$. https://doi.org/10.1016/j.cognition.2007.04.004.

Lenth, R. (2020). emmeans: Estimated Marginal Means, aka Least-Squares Means. R package version 1.5.0.

Leslie, A. M. (1984). Spatiotemporal continuity and the perception of causality in infants. Perception, 13, 287-305. https://doi. org/10.1068/p130287.

Leslie, A. M. (1993). A theory of agency. In D. Sperber, D. Premack, \& A. J. Premack (Eds.), Causal cognition: A multidisciplinary debate (pp. 121-141). Oxford, UK: Oxford University Press..

Leslie, A. M., \& Keeble, S. (1987). Do six-month-old infants perceive causality? Cognition, 25, 265-288. https://doi.org/10.1016/ S0010-0277(87)80006-9.

Lidz, J., Gleitman, H., \& Gleitman, L. (2003). Understanding how input matters: Verb learning and the footprint of universal grammar. Cognition, 87, 151-178. https://doi.org/10.1016/S0010-0277(02)00230-5.

Marchman, V. A., \& Bates, E. (1994). Continuity in lexical and morphological development: A test of the critical mass hypothesis. Journal of Child Language, 21(2), 339-366. https://doi.org/10.1017/S0305000900009302.

Meltzoff, A. N., Waismeyer, A., \& Alison, G. (2012). Learning about causes from people: Observational causal learning in $24-$ month-old infants. Developmental Psychology, 48, 1215-1228. https://doi.org/10.1037/a0027440.

Michotte, A. (1963). The perception of causality. New York: Basic Books..

Muentener, P., Bonawitz, E., Horowitz, A., \& Schulz, L. (2012). Mind the gap: Investigating toddlers' sensitivity to contact relations in predictive events. PLOS ONE, 7(4), e34061. https://doi.org/10.1371/journal.pone.0034061. 
Muentener, P., \& Carey, S. (2010). Infants' causal representations of state change events. Cognitive Psychology, 61, 63-86. https:// doi.org/10.1016/j.cogpsych.2010.02.001.

Naigles, L. R. (1990). Children use syntax to learn verb meanings. Journal of Child Language, 17, 357-374. https://doi.org/10.1017/ S0305000900013817.

Nazzi, T., \& Gopnik, A. (2000). A shift in children's use of perceptual and causal cues to categorization. Developmental Science, 3 , 389-396. https://doi.org/10.1111/1467-7687.00133.

Nelson, K. (2005). Language pathways into the community of minds. In J. W. Astington \& J. A. Baird (Eds.), Why language matters for theory of mind (pp. 26-49). Oxford, UK: Oxford University Press..

Peirce, J., \& MacAskill, M. (2018). Building experiments in PsychoPy. Thousand Oaks, CA: Sage.

R Core Team (2020). R: A language and environment for statistical computing. Vienna, Austria: R Foundation for Statistical Computing. https://www.R-project.org..

Saxe, R., Tenenbaum, J., \& Carey, S. (2005). Secret agents: Inferences about hidden causes by 10- and 12-month-old infants. Psychological Science, 16, 995-1001. https://doi.org/10.1111/j.1467-9280.2005.01649.x.

Saxe, R., Tzelnic, T., \& Carey, S. (2007). Knowing who dunnit: Infants identify the causal agent in an unseen causal interaction. Developmental Psychology, 43, 149-158. https://doi.org/10.1037/0012-1649.43.1.149.

Schulz, L. E., Bonawitz, E. B., \& Griffiths, T. L. (2007). Can being scared cause tummy aches? Naive theories, ambiguous evidence, and preschoolers' causal inferences. Developmental Psychology, 43, 1124-1139. https://doi.org/10.1037/00121649.43.5.1124.

Sloetjes, H., \& Wittenburg, P. (2008). Annotation by category: ELAN and ISO DCR. In N. Calzolari, K. Choukri, B. Maegaard, J. Mariani, J. Odijk, S. Piperidis, \& D. Tapias (Eds.), Proceedings of the Sixth International Conference on Language Resources and Evaluation (LREC 2008). Paris: European Language Resources Association.

Shibatani, M., \& Pardeshi, P. (2002). The causative continuum. Typological Studies in Language, 48, 85-126. https://doi.org/ $10.1075 /$ tsl.48.07shi.

Woodward, J. (2007). Interventionist theories of causation in psychological perspective. In A. Gopnik \& L. Schulz (Eds.), Causal learning: Psychology, philosophy, and computation (pp. 19-36). New York: Oxford University Press. https://doi.org/10.1093/ acprof:oso/9780195176803.003.0002. 